\title{
Implementation of Kane's Method for a Spacecraft Composed of Multiple Rigid Bodies
}

\author{
Eric T. Stoneking * \\ NASA Goddard Space Flight Center, Greenbelt, MD 20771, USA
}

\begin{abstract}
Equations of motion are derived for a general spacecraft composed of rigid bodies connected via rotary (spherical or gimballed) joints in a tree topology. Several supporting concepts are developed in depth. Basis dyads aid in the transition from basis-free vector equations to component-wise equations. Joint partials allow abstraction of 1-DOF, 2-DOF, 3-DOF gimballed and spherical rotational joints to a common notation. The basic building block consisting of an "inner" body and an "outer" body connected by a joint enables efficient organization of arbitrary tree structures. Kane's equation is recast in a form which facilitates systematic assembly of large systems of equations, and exposes a relationship of Kane's equation to Newton and Euler's equations which is obscured by the usual presentation. The resulting system of dynamic equations is of minimum dimension, and is suitable for numerical solution by computer. Implementation is discussed, and illustrative simulation results are presented.
\end{abstract}

\section{Nomenclature}

Reference Frames and Points

$N \quad$ A Newtonian (non-accelerating, non-rotating) frame

$B \quad$ A Body frame

$B_{j}$ The $j$ th Body frame

$B_{j}^{*} \quad$ Mass center of $B_{j}$

$G_{k} \quad$ The $k$ th Joint

$B_{i} \quad$ Inner Body

$B_{o} \quad$ Outer Body

Vector-Dyad Notation

$\vec{x} \quad$ A vector

$x_{j} \quad$ The components of $\vec{x}$ in frame $B_{j}$

$\underline{\underline{B}}$ The Basis Dyad for frame $B$

$\underline{\Omega}$ Partial Angular Velocity Dyad

$\underline{\underline{\omega}} \quad$ Partial Velocity Dyad

$\vec{\omega} \quad$ Angular velocity of $B$ in $N$

$\vec{v} \quad$ Linear velocity of $B^{*}$ in $N$

$\hat{b}_{k} \quad$ Orthonormal basis vectors for $B,(k=1,2,3)$

$\hat{b}_{j k} \quad$ Orthonormal basis vectors for $B_{j},(k=1,2,3)$

$\hat{n}_{k} \quad$ Orthonormal basis vectors for $N,(k=1,2,3)$

$\vec{r}_{j k} \quad$ The path vector from $B_{j}^{*}$ to joint $G_{k}$

$\vec{\beta}_{j} \quad$ The path vector from $B_{j}^{*}$ to $B_{1}^{*}$

Matrix Notation

$U \quad$ Identity Matrix

$\Omega \quad$ Partial Angular Velocity Matrix

$V \quad$ Partial Velocity Matrix

*Aerospace Engineer, Code 591, AIAA Member. 
Dynamic states (generalized speeds)

$x \quad$ Kinematic states (generalized coordinates)

$\omega$ Components of $\vec{\omega}$, expressed in $B$

$v \quad$ Components of $\vec{v}$, expressed in $N$

$\theta \quad$ Gimbal angles

$\sigma \quad$ Joint angular rates

$\Gamma \quad$ Joint partial matrix

$H$ Angular momentum

$m \quad$ Mass

I Central Moments of Inertia

F Force

$T$ Torque

a Acceleration

$\alpha \quad$ Angular Acceleration

$a_{r} \quad$ Remainder acceleration

$\alpha_{r} \quad$ Remainder angular acceleration

${ }^{j} C^{k}$ Direction Cosine Matrix between frames $B_{j}$ and $B_{k}$

$r^{\times} \quad$ The skew-symmetric matrix such that $r^{\times} x=\vec{r} \times \vec{x}$

\section{Introduction}

A broad range of spacecraft may be well modeled as a collection of rigid bodies connected in a tree topology by rotational joints. The complexity of appropriate dynamical models can quickly outstrip the limited examples available in textbooks. Powerful and general commercial dynamics software packages are available, but require an investment of money and training time, and present the challenge of integrating their dynamics capabilities with custom environmental and control models. Consequently this practitioner, like many before, undertook to develop custom simulation code to solve the range of problems that we face in our daily work. The work presented here is one practitioner's journey from textbook to working simulation code. There is no "new" theory introduced, but we emphasize some concepts that we found key to successful application. The objective is to obtain a system of differential equations of motion amenable to solution by numerical integration. We talk about Kane's equation, degrees of freedom and constraints, basis dyads, partial velocities and joint partials. We focus on the building block composed of a single joint connecting an "inner" and "outer" body, then use that to assemble trees of arbitrary complexity. We present a top-level algorithm of our simulation code, and present some illustrative simulation results.

\section{Kane's Equation}

Kane's equation is presented as a set of scalar equations: ${ }^{1}$

$$
F_{r}+F_{r}^{*}=0, \quad r=1, \ldots, n
$$

where $n$ is the number of degrees of freedom of the system. For a system comprising a set of $N_{b}$ rigid bodies, the generalized active forces are given by

$$
F_{r}=\sum_{k=1}^{N_{b}}\left(\vec{\omega}_{r}^{k} \cdot \vec{T}_{k}\right)+\sum_{k=1}^{N_{b}}\left(\vec{v}_{r}^{k} \cdot \vec{F}_{k}\right)
$$

and the generalized inertia forces are given by

$$
F_{r}^{*}=\sum_{k=1}^{N_{b}}\left[\vec{\omega}_{r}^{k} \cdot\left(-I_{k} \vec{\alpha}_{k}-\vec{\omega}_{k} \times \vec{H}_{k}\right)\right]+\sum_{k=1}^{N_{b}}\left[\vec{v}_{r}^{k} \cdot\left(-m_{k} \vec{a}_{k}\right)\right]
$$

Regrouping, we may write Kane's equation in the form

$$
\sum_{k=1}^{N_{b}}\left[\vec{\omega}_{r}^{k} \cdot(\vec{T}-I \vec{\alpha}-\vec{\omega} \times \vec{H})_{k}\right]+\sum_{k=1}^{N_{b}}\left[\vec{v}_{r}^{k} \cdot(\vec{F}-m \vec{a})_{k}\right]=0
$$


The expressions in parentheses are identically zero, being Euler's equation and Newton's second law of motion for each body in the system. The vector quantities $\vec{\omega}_{r}$ and $\vec{v}_{r}$ are the partial angular velocity and partial velocity, respectively. From a purely formal point of view, it doesn't matter what $\vec{\omega}_{r}$ and $\vec{v}_{r}$ are; the equation is satisfied given any bounded functions. We will discuss shortly the partial velocities in more detail. First, we would like to motivate that discussion by discussing the system of rigid bodies, and the system of equations we need to describe their motion.

\section{How Many Equations?}

A single rigid body has six degrees of freedom - three rotational and three translational. A collection of $N_{b}$ rigid bodies may have as many as $6 N_{b}$ degrees of freedom, requiring up to $6 N_{b}$ differential equations of motion to solve. For the problems that interest us, however, we connect the rigid bodies with rotary joints. Each joint may have one, two, or three rotational degrees of freedom and no translational degrees of freedom. Said another way, each rotary joint introduces three translational constraints and zero, one, or two rotational constraints. If $N_{c}$ is the total number of constraints, then there are $N_{u}=6 N_{b}-N_{c}$ degrees of freedom remaining.

There are several methods that we could apply to solve for the motion of the system. One method is to use Euler's and Newton's equations for each body individually, each being acted upon by the constraint forces (and possibly torques) arising from the joint constraints. This method requires $6 N_{b}$ dynamical states (generalized speeds), or alternatively, $N_{u}$ generalized speeds and $N_{c}=6 N_{b}-N_{u}$ constraint forces and torques. The joint constraints may be expressed as differential equations, ${ }^{5}$ or as algebraic equations. ${ }^{6}$ Either way, they must be solved simultaneously with the dynamics equations, yielding a total system size of $6 N_{b}$ equations.

Computational speed and numerical accuracy are both good reasons to look for a minimum-dimension system of equations. The computation time required to solve a system of equations scales as the square of the dimension of the system, and the forces and torques required to enforce a joint constraint may be quite large, driving the numerical integration timestep to be very small to keep the solution from diverging. We are generally not interested in the constraint forces and torques, so we seek a formulation that does not require solving for them.

Kane's method (among others) arrives at the minimum-dimension system of equations by careful choice of dynamical variables, and by not requiring solution of the constraint equations. By assigning as many independent generalized speeds as there are degrees of freedom, we partition the $6 N_{b}$-dimensional space of system motions into two disjoint subspaces: the $N_{u^{-}}$-dimensional space of permissible motions, and the $N_{c^{-}}$ dimensional space of impermissible motions. All we need is something that projects Euler's and Newton's equations into that permissible subspace, and the constraints are automatically eliminated. Looking back at equation (4), it is precisely the partial angular velocities and partial velocities that perform that projection.

Should it be desired to find some constraint forces or torques after all, it is possible to do so by application of the principle of virtual power. The reader may have been exposed to the principle of virtual work: by assuming an infinitesimal virtual (i.e. impermissible) motion, one may find the associated constraint equation required to enforce that the virtual motion is zero. The principle of virtual power is the principle that underlies all of Kane's method, and it is really the same technique, using a virtual generalized speed instead of a virtual motion (power being the time derivative of work). By introducing, for example, three generalized speeds for the translational motion of a rotary joint, we may find the partial velocities and formulate equations of motion. Once the equations of motion are obtained, we may set the virtual generalized speeds (and their derivatives) to zero analytically. The virtual speeds will thus be eliminated from the equations of motion, restoring the minimum-dimension set of equations, and a set of three additional equations will be obtained expressing the desired translational constraints. We won't discuss this technique further in this paper - we only mention it to emphasize the division of the motion space into permissible and impermissible (constraint) subspaces. The usual selection of generalized speeds spans the subspace of permissible motions, saving the labor of solving constraints. But we can access the constraint subspace if desired. 


\section{Basis Dyads, Partial Velocities and Generalized Speeds}

Consider a single rigid body. We may write the angular velocity of the body, $\vec{\omega}$, and the linear velocity of the body's mass center, $\vec{v}$, in terms of six generalized speeds:

$$
\begin{aligned}
\vec{\omega} & =u_{1} \hat{b}_{1}+u_{2} \hat{b}_{2}+u_{3} \hat{b}_{3} \\
\vec{v} & =u_{4} \hat{n}_{1}+u_{5} \hat{n}_{2}+u_{6} \hat{n}_{3}
\end{aligned}
$$

We may write this more compactly by introducing basis $d y a d s^{\mathbf{a}}$,

$$
\begin{aligned}
\underline{\underline{B}} & \equiv\left[\begin{array}{lll}
\hat{b}_{1} & \hat{b}_{2} & \hat{b}_{3}
\end{array}\right] \\
\underline{\underline{\underline{N}}} & \equiv\left[\begin{array}{lll}
\hat{n}_{1} & \hat{n}_{2} & \hat{n}_{3}
\end{array}\right]
\end{aligned}
$$

so that

$$
\begin{aligned}
\vec{\omega} & =\underline{\underline{B}} u_{1: 3} \\
\vec{v} & =\underline{\underline{N}} u_{4: 6}
\end{aligned}
$$

Concatenating the angular and linear generalized speeds,

$$
\begin{aligned}
\vec{\omega} & =\underline{\underline{\Omega}} u \\
\vec{v} & =\underline{\underline{V}} u
\end{aligned}
$$

where $\underline{\underline{\Omega}}=[\underline{\underline{B}} 0]$ and $\underline{\underline{V}}=[0 \underline{\underline{N}}]$. These dyadics are simply a compact way to express the partial angular velocities and partial velocities. For a single rigid body, the extra notation is hardly worth it. But consider now a system of two (uncoupled) rigid bodies, and write expressions for their angular velocities and the linear velocities of their mass centers:

$$
\begin{aligned}
& \vec{\omega}_{1}=u_{1} \hat{b}_{11}+u_{2} \hat{b}_{12}+u_{3} \hat{b}_{13} \\
& \vec{\omega}_{2}=u_{4} \hat{b}_{21}+u_{5} \hat{b}_{22}+u_{6} \hat{b}_{23} \\
& \vec{v}_{1}=u_{7} \hat{n}_{1}+u_{8} \hat{n}_{2}+u_{9} \hat{n}_{3} \\
& \vec{v}_{2}=u_{10} \hat{n}_{1}+u_{11} \hat{n}_{2}+u_{12} \hat{n}_{3}
\end{aligned}
$$

Using the basis dyad notation,

$$
\left\{\begin{array}{c}
\vec{\omega}_{1} \\
\vec{\omega}_{2} \\
\vec{v}_{1} \\
\vec{v}_{2}
\end{array}\right\}=\left[\begin{array}{cccc}
\underline{\underline{B}}_{1} & 0 & 0 & 0 \\
0 & \underline{\underline{B}} & 0 & 0 \\
0 & 0 & \underline{\underline{N}} & 0 \\
0 & 0 & 0 & \underline{\underline{N}}
\end{array}\right]\left\{\begin{array}{c}
u_{1: 3} \\
u_{4: 6} \\
u_{7: 9} \\
u_{10: 12}
\end{array}\right\}=[\underline{\underline{\underline{\Omega}}} \underline{\underline{\underline{V}}}] u
$$

where in this case

$$
\begin{aligned}
& \underline{\underline{\Omega}}=\left[\begin{array}{cccc}
\underline{\underline{B}}_{1} & 0 & 0 & 0 \\
0 & \underline{\underline{B}}_{2} & 0 & 0
\end{array}\right] \\
& \underline{\underline{V}}=\left[\begin{array}{llll}
0 & 0 & \underline{\underline{N}} & 0 \\
0 & 0 & 0 & \underline{\underline{N}}
\end{array}\right] .
\end{aligned}
$$

For any system the partial angular velocities, $\underline{\underline{\Omega}}$, are the partials of the relevant angular velocities, $\vec{\omega}$, with respect to the generalized speeds, $u$. Likewise, the partial velocities, $\underline{\underline{V}}$, are the partials of the relevant linear velocities, $\vec{v}$, with respect to $u$. For the systems under consideration, the relevant quantities are the angular velocities of the rigid bodies and the linear velocities of their mass centers.

As we move from formulation to implementation, we will want to shift from vector-dyad notation to matrix notation, eg. from $\vec{\omega}=\underline{\underline{\Omega}} u$ to $\omega=\Omega u$. To do this, we will use the relation between the direction cosine matrix relating two frames and their basis dyads:

$$
{ }^{B} C^{A}=\underline{\underline{B}}^{T} \underline{\underline{A}}
$$

${ }^{\text {a }}$ Hughes ${ }^{3}$ calls these vectrices. 
Vectors and their components may also be compactly related through basis dyads. For example, let $\omega_{k}=$ $\left[\begin{array}{lll}\omega_{k 1} & \omega_{k 2} & \omega_{k 3}\end{array}\right]$ be the components of $\vec{\omega}_{k}$ in $B_{k}$, and let $v_{k}=\left[\begin{array}{lll}v_{k 1} & v_{k 2} & v_{k 3}\end{array}\right]$ be the components of $\vec{v}_{k}$ in $N$. We may then write

$$
\begin{aligned}
\vec{\omega}_{k} & =\underline{\underline{B}}_{k} \omega_{k} \\
\vec{v}_{k} & =\underline{\underline{N}} v_{k}
\end{aligned}
$$

\section{Joint Partials}

A joint imposes a set of kinematic constraints on the motion of the two bodies that it connects. For this discussion, we limit our consideration to rotary joints: gimballed joints with one, two, or three degrees of freedom, as well as spherical (ball-and-socket) joints. We need a concise and general notation to represent all of these. We introduce this notation by example. Consider the rotation from a body $A$ to a body $B$ as a 3-DOF gimballed joint described by a Body-3, 3-1-2 Euler rotation through angles $\left(\theta_{1}, \theta_{2}, \theta_{3}\right)$. We write the relative angular velocity of $B$ with respect to $A$ in terms of the generalized speeds associated with the joint, $\sigma_{i} \equiv \dot{\theta}_{i}$ :

$$
A_{\vec{\omega}^{B}}=\underline{\underline{B}} \underbrace{\left[\begin{array}{ccc}
\cos \theta_{2} \sin \theta_{3} & \cos \theta_{3} & 0 \\
\cos \theta_{2} \cos \theta_{3} & -\sin \theta_{3} & 0 \\
-\sin \theta_{2} & 0 & 1
\end{array}\right]}_{\Gamma} \underbrace{\left\{\begin{array}{l}
\sigma_{1} \\
\sigma_{2} \\
\sigma_{3}
\end{array}\right\}}_{\sigma}
$$

The appearance of $\underline{\underline{B}}$ signifies that the product $\Gamma \sigma$ gives the components of ${ }^{A} \vec{\omega}^{B}$ expressed in $B$. Analogous to the partial angular velocities, the matrix $\Gamma$ relates the relative angular velocity of a joint to the joint's generalized speeds. We call this the matrix of joint partials. Later in the development, we will find that we need the time derivative of the joint partials, $\dot{\Gamma}$, in addition to $\Gamma$ itself.

Each Euler rotation sequence has a distinct joint partial matrix, with as many columns as the joint has degrees of freedom. For example, a Body-2, 2-3 Euler rotation through $\left(\theta_{1}, \theta_{2}\right)$ has $\sigma=\left[\sigma_{1} \sigma_{2}\right]$, and

$$
\Gamma=\left[\begin{array}{cc}
\sin \theta_{2} & 0 \\
\cos \theta_{2} & 0 \\
0 & 1
\end{array}\right], \quad \dot{\Gamma}=\left[\begin{array}{cc}
\sigma_{2} \cos \theta_{2} & 0 \\
-\sigma_{2} \sin \theta_{2} & 0 \\
0 & 0
\end{array}\right]
$$

A tabulation of $\omega(\dot{\theta})$ relations for all Euler rotation sequences may be found in reference. ${ }^{2}$ The joint partials are simply those relations in matrix form, and $\dot{\Gamma}$ is readily found by differentiation.

\section{Two Bodies and a Joint}

We restrict our study to systems of rigid bodies connected in a tree topology, ensuring that we can choose as many independent generalized speeds as there are degrees of freedom. In organizing the tree structure, we conceptualize a building block consisting of two bodies connected by a joint. While one body may have multiple "children", each joint has exactly one inner body and one outer body. This concept facilitates writing important kinematic and dynamic properties of the outer body $\left(B_{o}\right)$ in terms of the properties of the inner body $\left(B_{i}\right)$ and the joint $\left(G_{k}\right)$. The angular velocity of the outer body is constructed as

$$
\begin{aligned}
{ }^{N} \vec{\omega}^{o} & ={ }^{N} \vec{\omega}^{i}+{ }^{i} \vec{\omega}^{o} \\
& =\underline{\underline{B}}_{i} \omega_{i}+\underline{\underline{B}}_{0} \Gamma_{k} \sigma_{k}
\end{aligned}
$$

The velocity of the outer body's mass center may be written

$$
\begin{aligned}
{ }^{N} \vec{v}^{o} & ={ }^{N} \vec{v}^{i}+{ }^{N} \vec{\omega}^{i} \times \vec{r}_{i k}-{ }^{N} \vec{\omega}^{o} \times \vec{r}_{o k} \\
& =\underline{\underline{N}} v_{i}+\left(\underline{\underline{B}}_{i} \omega_{i}\right) \times\left(\underline{\underline{N}} r_{i k}-\underline{\underline{N}} r_{o k}\right)-\left(\underline{\underline{B}}_{o} \Gamma_{k} \sigma_{k}\right) \times\left(\underline{\underline{N}} r_{o k}\right)
\end{aligned}
$$

where $\vec{r}_{i k}$ and $\vec{r}_{o k}$ are the vectors from the bodies' respective mass centers to the joint.

It is natural to express some vector quantities in terms of their components in one frame or another (eg. $\left.\underline{\underline{N}} v_{i}, \underline{\underline{B}}_{i} \omega_{i}\right)$. It is not obvious yet which frame is most natural to express the $\vec{r}$ 's. We choose $N$ in 
anticipation of more complex systems; differences of vectors, each fixed in a different body, are expressed in $N$ with equal inconvenience.

The dynamical state vector for this two-body system is the concatenation of the inner body's angular velocity components, the generalized speeds associated with the joint, and the components of the velocity of the mass center of the inner body expressed in $N: u=\left[\begin{array}{lll}\omega_{1} & \sigma_{1} & v_{1}\end{array}\right]^{T}$. Writing the angular velocities and linear velocities for the system:

$$
\begin{aligned}
& \left\{\begin{array}{l}
\vec{\omega}_{1} \\
\vec{\omega}_{2}
\end{array}\right\}=\left[\begin{array}{ccc}
\underline{\underline{B}}_{1} & 0 & 0 \\
\underline{\underline{B}}_{1} & \underline{\underline{B}}_{2} \Gamma_{1} & 0
\end{array}\right]\left\{\begin{array}{l}
\omega_{1} \\
\sigma_{1} \\
v_{1}
\end{array}\right\} \\
& \left\{\begin{array}{l}
\vec{v}_{1} \\
\vec{v}_{2}
\end{array}\right\}=\left[\begin{array}{ccc}
0 & 0 & \underline{\underline{N}} \\
\left(\underline{\underline{N}} r_{21}-\underline{\underline{N}} r_{11}\right)^{\times} \underline{\underline{B}}_{1} & \left(\underline{\underline{N}} r_{21}\right)^{\times} \underline{\underline{B}}_{2} \Gamma_{1} & \underline{\underline{N}}
\end{array}\right]\left\{\begin{array}{l}
\omega_{1} \\
\sigma_{1} \\
v_{1}
\end{array}\right\}
\end{aligned}
$$

We may clear the basis dyads by multiplying the $\omega_{1}$ row by $\underline{\underline{B}}_{1}^{T}$, the $\omega_{2}$ row by $\underline{\underline{B}}_{2}^{T}$, and the $v$ rows by $\underline{\underline{N}}^{T}$ :

$$
\begin{aligned}
& \left\{\begin{array}{l}
\omega_{1} \\
\omega_{2}
\end{array}\right\}=\underbrace{\left[\begin{array}{ccc}
U & 0 & 0 \\
{ }^{2} C^{1} & \Gamma_{1} & 0
\end{array}\right]}_{\Omega}\left\{\begin{array}{l}
\omega_{1} \\
\sigma_{1} \\
v_{1}
\end{array}\right\} \\
& \left\{\begin{array}{l}
v_{1} \\
v_{2}
\end{array}\right\}=\underbrace{\left[\begin{array}{ccc}
0 & 0 & U \\
\beta_{2}^{\times N} C^{1} & r_{21}^{\times} C^{2} \Gamma_{1} & U
\end{array}\right]}_{V}\left\{\begin{array}{l}
\omega_{1} \\
\sigma_{1} \\
v_{1}
\end{array}\right\}
\end{aligned}
$$

where we've introduced $\beta_{2} \equiv r_{21}-r_{11}$ for brevity.

The partial velocity matrices $\Omega$ and $V$ contain configuration-related quantities that will have to be evaluated at each evaluation of the equations of motion. This aside, the system velocities (angular and linear) are linear functions of the generalized speeds:

$$
\begin{aligned}
& \{\omega\}=\Omega u \\
& \{v\}=V u
\end{aligned}
$$

We present a five-body example in the appendix, to highlight the patterns in the structure of $\Omega$ and $V$ that arise. Using these patterns, partial velocities for arbitrary tree structures may be constructed by inspection.

Carrying on, we find the outer body's angular acceleration,

$$
{ }^{N} \vec{\alpha}^{o}={ }^{N} \vec{\alpha}^{i}+\underline{\underline{B}}_{o}\left(\Gamma_{k} \dot{\sigma}_{k}+\dot{\Gamma}_{k} \sigma_{k}+\omega_{o} \times \Gamma_{k} \sigma_{k}\right)
$$

and the linear acceleration of its mass center:

$$
\begin{aligned}
{ }^{N} \vec{a}^{o}= & { }^{N} \vec{a}^{i}+{ }^{N} \vec{\omega}^{i} \times\left({ }^{N} \vec{\omega}^{i} \times \vec{r}_{i k}\right)+{ }^{N} \vec{\alpha}^{i} \times \vec{r}_{i k}-{ }^{N} \vec{\omega}^{o} \times\left({ }^{N} \vec{\omega}^{o} \times \vec{r}_{o k}\right)-{ }^{N} \vec{\alpha}^{o} \times \vec{r}_{o k} \\
= & { }^{N} \vec{a}^{i}+{ }^{N} \vec{\omega}^{i} \times\left({ }^{N} \vec{\omega}^{i} \times \vec{r}_{i k}\right)+{ }^{N} \vec{\alpha}^{i} \times \vec{r}_{i k}-\left({ }^{N} \vec{\omega}^{i}+\underline{\underline{B}}_{o} \Gamma_{k} \sigma_{k}\right) \times\left[\left({ }^{N} \vec{\omega}^{i}+\underline{\underline{B}}_{o} \Gamma_{k} \sigma_{k}\right) \times \vec{r}_{o k}\right] \\
& -\left[{ }^{N} \vec{\alpha}^{i}+\underline{\underline{B}}_{o}\left(\Gamma_{k} \dot{\sigma}_{k}+\dot{\Gamma}_{k} \sigma_{k}+\omega_{o} \times \Gamma_{k} \sigma_{k}\right)\right] \times \vec{r}_{o k}
\end{aligned}
$$

Assembling these for the system, we find that there are terms that include $\dot{u}$ and terms that don't. We coin the term remainder accelerations for the latter, and denote them by $\alpha_{r}$ and $a_{r}$. They may be constructed recursively in the same fashion as $\alpha$ and $a$ :

$$
\begin{aligned}
& { }^{N} \vec{\alpha}_{r}^{o}={ }^{N} \vec{\alpha}^{i}{ }_{r}+\underline{\underline{B}}_{o}\left(\dot{\Gamma}_{k} \sigma_{k}+\omega_{o} \times \Gamma_{k} \sigma_{k}\right) \\
& { }^{N} \vec{a}_{r}^{o}={ }^{N} \vec{a}_{r}^{i}{ }_{r}+{ }^{N} \vec{\omega}^{i} \times\left({ }^{N} \vec{\omega}^{i} \times \vec{r}_{i k}\right)+{ }^{N} \vec{\alpha}_{r}^{i} \times \vec{r}_{i k}-{ }^{N} \vec{\omega}^{o} \times\left({ }^{N} \vec{\omega}^{o} \times \vec{r}_{o k}\right)-{ }^{N} \vec{\alpha}_{r}^{o} \times \vec{r}_{o k}
\end{aligned}
$$

The remainder accelerations of the root body are zero: ${ }^{N} \vec{\alpha}^{1}{ }_{r}={ }^{N} \vec{a}_{r}{ }_{r}=0$.

The remainder accelerations allow us to write the system accelerations compactly as:

$$
\begin{aligned}
& \{\alpha\}=\Omega \dot{u}+\left\{\alpha_{r}\right\} \\
& \{a\}=V \dot{u}+\left\{a_{r}\right\}
\end{aligned}
$$




\section{The Matrix Form of Kane's Equation}

Equation 4 gives Kane's equation in vector form. We have spent the intervening text developing matrix representations for the key factors in this equation. We have found matrix constructions for the partial velocities, angular accelerations and linear accelerations. The remaining factors appear in the sums over bodies - each body $B_{k}$ has a mass, $m_{k}$, a central inertia matrix, $I_{k}$, is acted on by active forces $F_{k}$ and torques $T_{k}$, and has at any instant a gyroscopic torque term, $(\omega \times H)_{k}$. Using $[m],[I],\{F\},\{T\}$, and $\{\omega \times H\}$ to denote the matrices formed by assembling these for the system, we may write Kane's equation in matrix form:

$$
\Omega^{T}(\{T\}-[I]\{\alpha\}-\{\omega \times H\})+V^{T}(\{F\}-[m]\{a\})=0
$$

Substituting equations (38)-(39), and grouping on $\dot{u}$, we obtain

$$
\left(\Omega^{T}[I] \Omega+V^{T}[m] V\right) \dot{u}=\Omega^{T}\left(\{T\}-[I]\left\{\alpha_{r}\right\}-\{\omega \times H\}\right)+V^{T}\left(\{F\}-[m]\left\{a_{r}\right\}\right)
$$

or, letting the brackets be implied by the context,

$$
\left(\Omega^{T} I \Omega+V^{T} m V\right) \dot{u}=\Omega^{T}\left(T-I \alpha_{r}-\omega \times H\right)+V^{T}\left(F-m a_{r}\right)
$$

\section{Kinematic Equations of Motion}

To this point, we have focused on the dynamic equations of motion, giving scant attention to the kinematic relationships. For the systems of interest, the kinematics are relatively simple. As the dynamic state vector is arranged by the generalized speeds, $u=\left[\omega_{1} \sigma v_{1}\right]^{T}$, we arrange the kinematic state vector by the generalized coordinates, $x=\left[\begin{array}{lll}q_{1} & \theta & p_{1}\end{array}\right]^{T}$.

For $B_{1}$, we parameterize attitude using a quaternion, ${ }^{1} q^{N}$. The rates of change of quaternion components are given by:

$$
\left\{\begin{array}{l}
\dot{q}_{1} \\
\dot{q}_{2} \\
\dot{q}_{3} \\
\dot{q}_{4}
\end{array}\right\}=\frac{1}{2}\left[\begin{array}{ccc}
q_{4} & -q_{3} & q_{2} \\
q_{3} & q_{4} & -q_{1} \\
-q_{2} & q_{1} & q_{4} \\
-q_{1} & -q_{2} & -q_{3}
\end{array}\right]\left\{\begin{array}{l}
\omega_{1} \\
\omega_{2} \\
\omega_{3}
\end{array}\right\}
$$

We have mentioned in passing the kinematic relation for a gimballed joint - representing the joint rotation by the Euler angles $\theta$, the kinematic relation is simply

$$
\dot{\theta}=\sigma
$$

Spherical joints are better represented using a quaternion for the joint rotation, ${ }^{\circ} q^{i}$, using (43).

The last generalized coordinate is the position of $B_{1}$ 's mass center in $N$, expressed in $N$. The kinematic relation is simply

$$
\dot{p}_{1}=v_{1}
$$

\section{Computer Implementation}

Equation (42) is a set of $N_{u}$ equations in the form

$$
[C O E F] \dot{u}=\{R H S\}
$$

The COEF matrix is $N_{u} \times N_{u}$, and $R H S$ is an $N_{u} \times 1$ array. This system is suitable for computer solution. The partial velocity matrices encapsulate all the coordinate transformations. Numerical solvers such as Cholesky decomposition exploit the symmetry of the COEF matrix, running about twice as fast as Gaussian elimination.

Numerical solvers ${ }^{\mathrm{b}}$ typically require multiple evaluations of the equations of motion over each integration timestep. To properly assemble the COEF and $R H S$, the entire system configuration must be calculated at each EOM evaluation. Our implementation follows the algorithm presented in Table 1.

\footnotetext{
bFor our applications, a fixed-step 4th-order Runge-Kutta works fine.
} 
Table 1. Algorithm for Evaluation of Equations of Motion

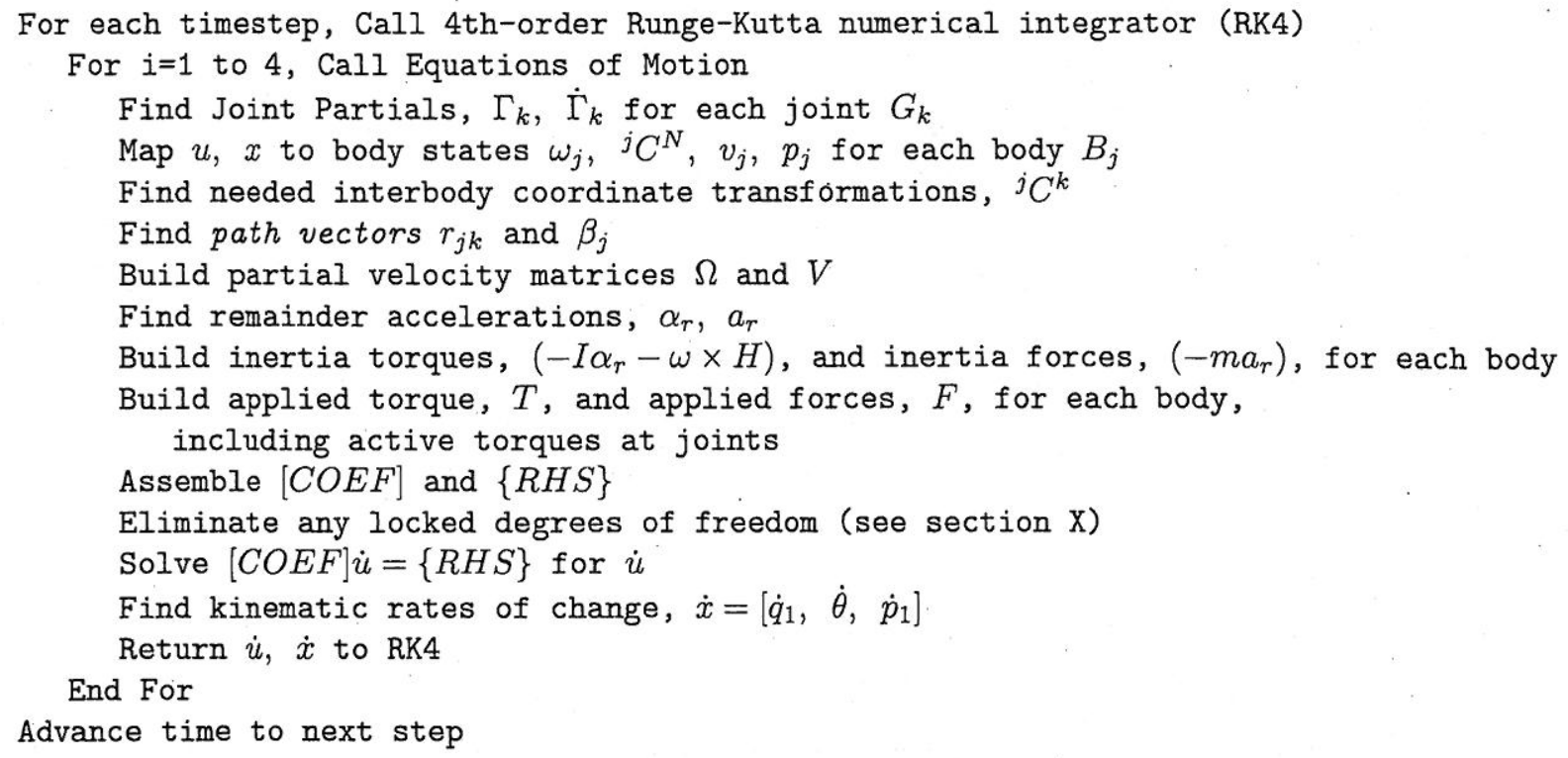

\section{Eliminating Degrees of Freedom}

Using the generalized speeds as our dynamic variables allows us to easily eliminate a degree of freedom from the equations of motion. Of course, if it weren't useful, we shouldn't have put it in the model to begin with, but there are situations where it may be desirable to temporarily lock out a degree of freedom for a specific simulation case, or even a portion of one. For example, one axis of a joint may be locked to model a parking brake on a robot arm, or to model a gimbal motor failure. The form of the equations of motion makes it straightforward to do this without otherwise changing the spacecraft model.

Consider the degree of freedom corresponding to $u_{k}$. Locking that DOF reduces the dimension of the subspace of permissible motions by one, consequently adding one equation into the subspace of constraints. We set $u_{k}=\dot{u}_{k}=0$, and the associated kinematic state, $x_{k}$, retains the value it had when the DOF became locked. The equations of motion are reduced by eliminating the $k$ th row and column of $[C O E F]$, and the $k$ th row of $\{R H S\}$.

\section{Illustrative Simulation Results}

To check our implementation, we will demonstrate a classic lesson from the dawn of spaceflight. Consider a spacecraft composed of a cylindrical body and four small appendages, as shown in figure 1. The spacecraft is spin-stabilized about its minor axis. Rotating as a solid body, linear perturbation theory shows that this is a stable motion. The experience of Explorer $\mathrm{I}^{7}$ demonstrates, however, that internal energy dissipation can destabilize a minor-axis spin in favor of a major-axis ("flat") spin, which has less energy for the same angular momentum. The internal energy dissipation was attributed to deflections of the turnstile antenna, excited by nutation.

We construct a five-body dynamical model, each appendage connected to the main body by a two-axis gimballed joint. We introduce a spring and damping torque in each joint. To accentuate the coupling, we deliberately tune the joint parameters to resonate with the nutation of the system.

The coupling is primarily in the joint axes that are perpendicular to the spin axis. When spinning about the major axis, only two of the joint DOFs are expected to interact strongly with nutation - we tune these two DOFs to resonate with the major-axis nutation frequency. The remaining six joint DOFs are tuned to resonate with the minor-axis nutation motion.

Figure 2 shows the time history of the angular rates of the central body, expressed in the body frame. Initialized with a steady spin of $5 \mathrm{deg} / \mathrm{sec}$ about the $\mathrm{Z}$ axis, and a very small transverse rate (1.0E-3 deg/sec 


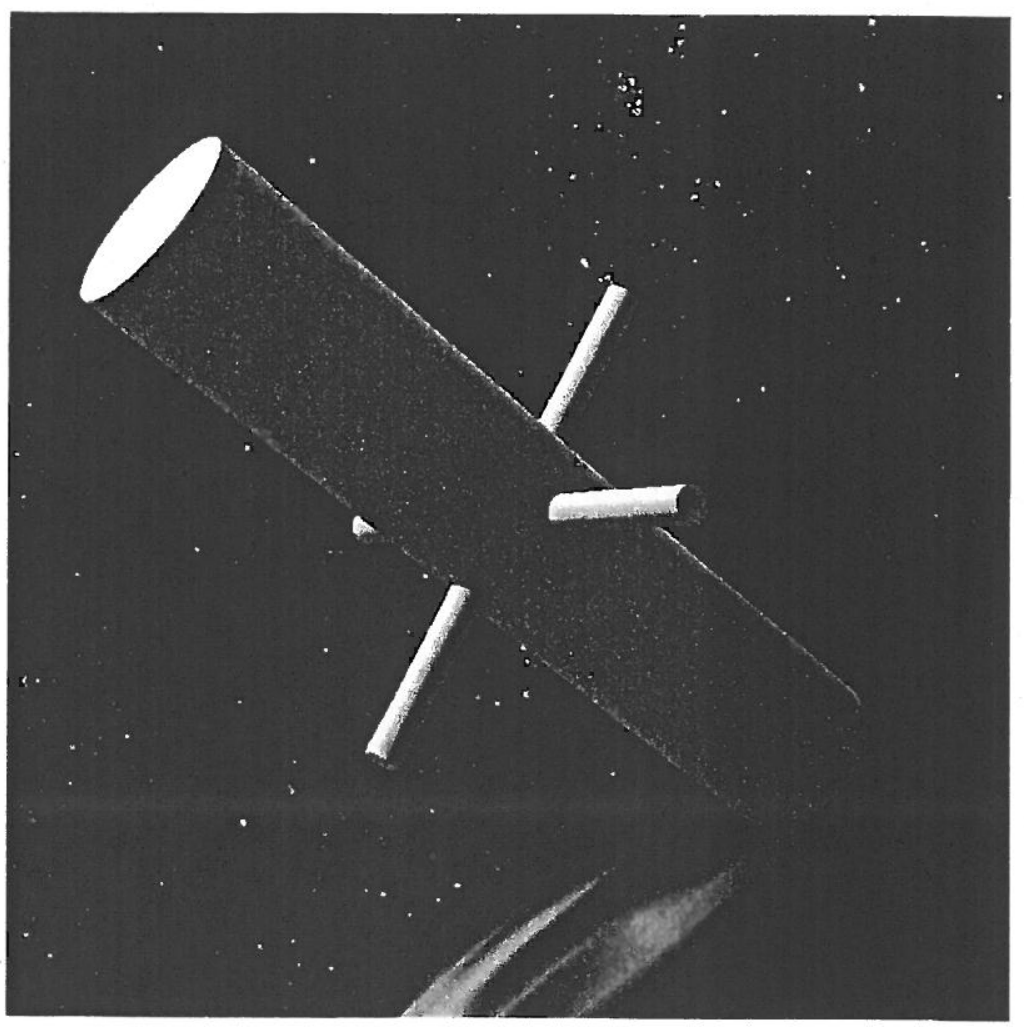

Figure 1. TumbleSat Model

about the $\mathrm{Y}$ axis), the nutation motion grows, eventually settling into a stable spin about the major (X) axis. Figure 3 shows the kinetic energy of the system, verifying that the major-axis spin is a lower-energy motion. The angular momentum was observed to be conserved, as expected. Not shown here, we repeated the simulation with all joint degrees of freedom locked, and observed that the minor-axis spin motion was stable under those conditions.

\section{Conclusion}

By recasting Kane's equations, we have shown that the partial velocities project Newton's Second Law into the subspace of permissible motions, and that the partial angular velocities do the same for Euler's equation. Our selection of generalized speeds is an implicit selection of which motions are permissible, and which aren't. Equations of motion and constraint equations are thus related, being disjoint subspaces that together comprise the total $6 N_{b}$-dimensional motion space. The same tools that we use to find the equations of motion may also be applied to find constraint equations or to eliminate a degree of freedom as desired.

We have used the joint-and-two-bodies building block to develop formulas that can be applied recursively over the system. We have used the concept of joint partials to handle all rotary joints with a common notation. The explicit use of basis dyads has helped us keep careful track of the relationships among the many reference frames involved while making the transition from vector-dyad notation to matrix notation.

We have emphasized implementation. For systems of this complexity, it does no good to have equations of motion if they aren't in a form you can solve. So we have presented not just the equations of motion but the algorithm for computing all the intermediate terms and assembling them for solution. The resulting system of differential equations is linear in the rates of change of the generalized speeds $(\dot{u})$, and is of minimum dimension.

We have limited our attention to a system of rigid bodies connected in a tree topology by rotary joints. This covers a broad range of spacecraft, and the concepts and notation developed here will be extended in future work to handle flexible bodies, translational joints, and (perhaps) more general topologies. 


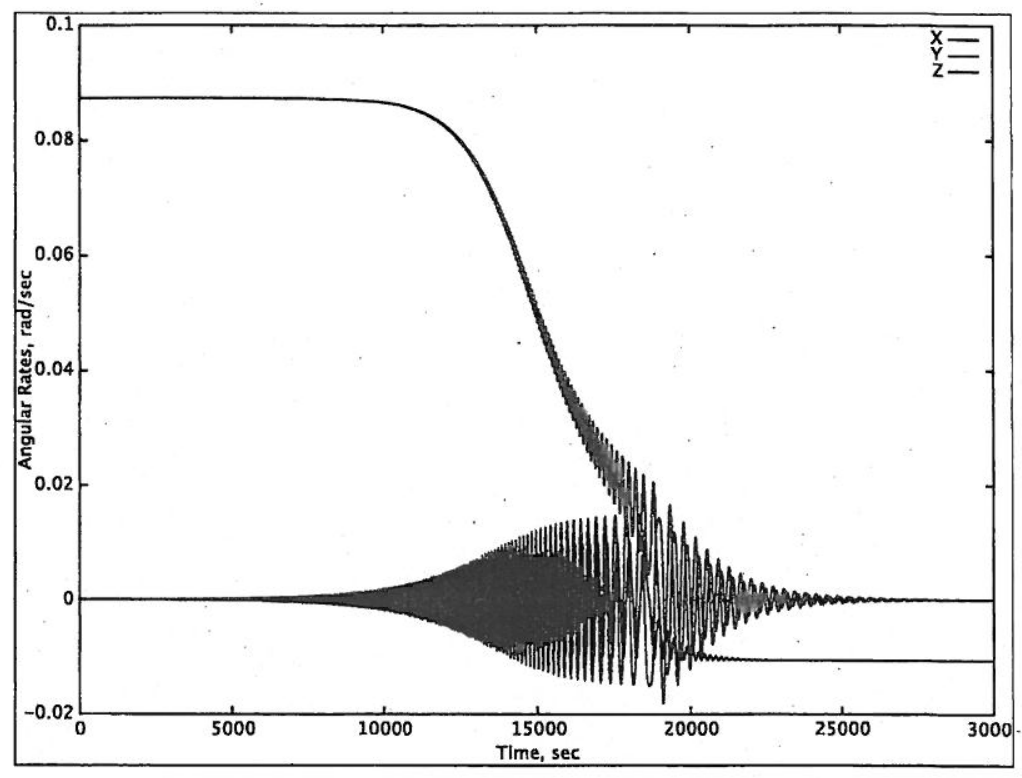

Figure 2. TumbleSat: Angular Velocity Components

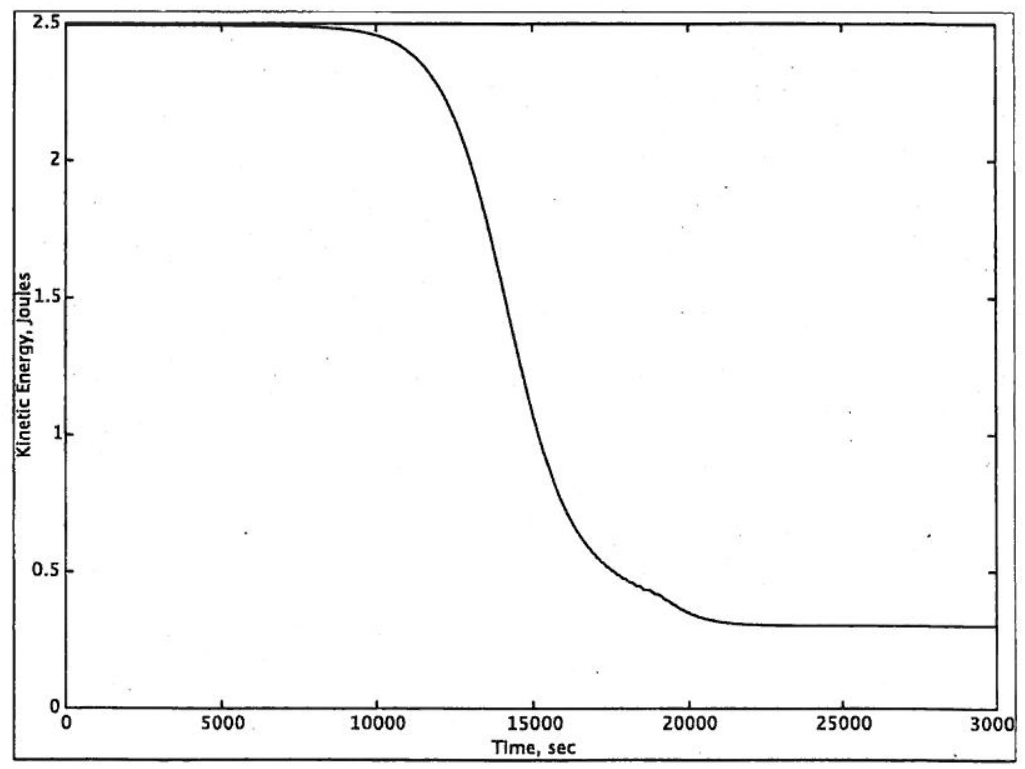

Figure 3. TumbleSat: Kinetic Energy 


\section{References}

${ }^{1}$ Kane, Thomas R. and Levinson, David A., Dynamics: Theory and Application, McGraw-Hill, 1985.

${ }^{2}$ Kane, Thomas R., Likins, Peter W., and Levinson, David A., Spacecraft Dynamics, McGraw-Hill, 1983.

${ }^{3}$ Hughes, Peter C., Spacecraft Attitude Dynamics, John Wiley and Sons, 1986.

${ }^{4}$ Amirouche, Farid, Fundamentals of Multibody Dynamics: Theory and Applications, Birkhäuser, 2006.

${ }^{5}$ Stoneking, Eric, "Newton-Euler Dynamic Equations of Motion for a Multi-body Spacecraft", Proceedings of 2007 AIAA GNC Conference, AIAA-2007-6441.

${ }^{6}$ Haug, Edward J., Computer-aided Kinematics and Dynamics of Mechanical Systems, Allyn and Bacon, 1989.

${ }^{7}$ Likins, P. W., "Spacecraft Attitude Dynamics and Control - A Personal Perspective on Early Developments," Journal of Guidance, Control, and Dynamics, Vol. 9, No. 2, 1986, pp. 129-134. 


\section{Appendix: Partial Velocity Matrices for a Five-Body Example}

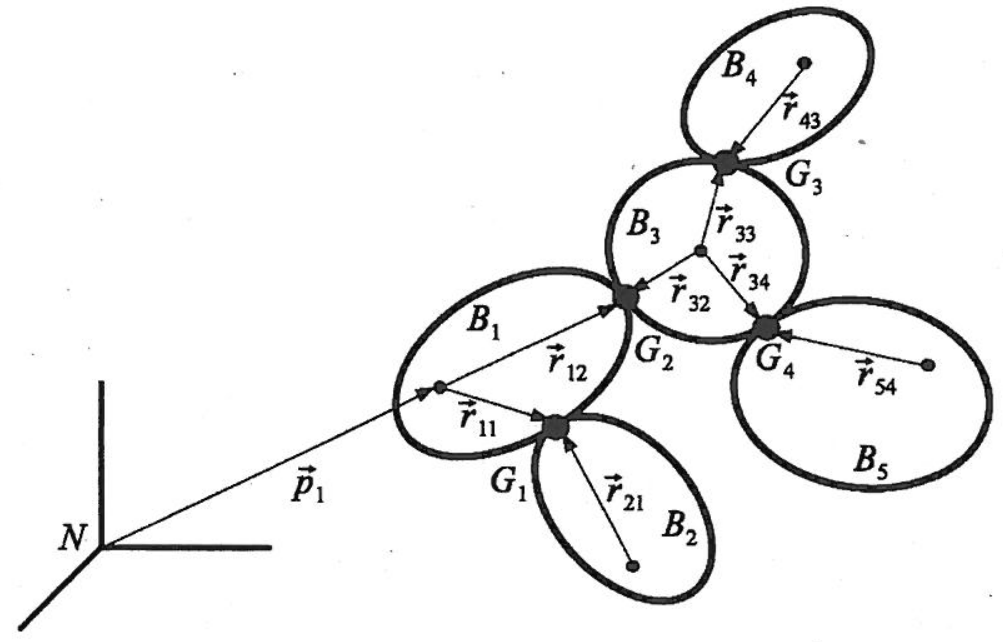

Figure 4. Five-Body Dynamical Model

To illustrate construction of the partial angular velocity and partial velocity matrices for a multi-body spacecraft, we consider the five-body model shown in figure 4. Using (25), we write the angular velocities of each body:

$$
\left\{\begin{array}{l}
\vec{\omega}_{1} \\
\vec{\omega}_{2} \\
\vec{\omega}_{3} \\
\vec{\omega}_{4} \\
\vec{\omega}_{5}
\end{array}\right\}=\left[\begin{array}{cccccc}
\underline{\underline{B}}_{1} & 0 & 0 & 0 & 0 & 0 \\
\underline{\underline{B}}_{1} & \underline{\underline{B}}_{2} \Gamma_{1} & 0 & 0 & 0 & 0 \\
\underline{\underline{B}}_{1} & 0 & \underline{\underline{B}}_{3} \Gamma_{2} & 0 & 0 & 0 \\
\underline{\underline{B}}_{1} & 0 & \underline{\underline{B}}_{3} \Gamma_{2} & \underline{\underline{B}}_{4} \Gamma_{3} & 0 & 0 \\
\underline{\underline{B}}_{1} & 0 & \underline{\underline{B}}_{3} \Gamma_{2} & 0 & \underline{\underline{B}}_{5} \Gamma_{4} & 0
\end{array}\right]\left\{\begin{array}{c}
\omega_{1} \\
\sigma_{1} \\
\sigma_{2} \\
\sigma_{3} \\
\sigma_{4} \\
v_{1}
\end{array}\right\}
$$

Clearing the basis dyads, we obtain

$$
\left\{\begin{array}{l}
\omega_{1} \\
\omega_{2} \\
\omega_{3} \\
\omega_{4} \\
\omega_{5}
\end{array}\right\}=\left[\begin{array}{cccccc}
U & 0 & 0 & 0 & 0 & 0 \\
{ }^{2} C^{1} & \Gamma_{1} & 0 & 0 & 0 & 0 \\
{ }^{3} C^{1} & 0 & \Gamma_{2} & 0 & 0 & 0 \\
{ }^{4} C^{1} & 0 & { }^{4} C^{3} \Gamma_{2} & \Gamma_{3} & 0 & 0 \\
{ }^{5} C^{1} & 0 & { }^{5} C^{3} \Gamma_{2} & 0 & \Gamma_{4} & 0
\end{array}\right]\left\{\begin{array}{c}
\omega_{1} \\
\sigma_{1} \\
\sigma_{2} \\
\sigma_{3} \\
\sigma_{4} \\
v_{1}
\end{array}\right\}
$$

Noting that the $j$ th row of $\Omega$ traces the path from $B_{1}$ to $B_{j}$, we conclude that we may write $\Omega$ for any tree-topology system by inspection.

Using (26), we write the linear velocities for the mass centers of each body:

$$
\left\{\begin{array}{l}
\vec{v}_{1} \\
\vec{v}_{2} \\
\vec{v}_{3} \\
\vec{v}_{4} \\
\vec{v}_{5}
\end{array}\right\}=\left[\begin{array}{cccccc}
0 & 0 & 0 & 0 & 0 & \underline{\underline{N}} \\
\left(\underline{\underline{N}} \beta_{2}\right)^{\times} \underline{\underline{B}}_{1} & \left(\underline{\underline{N}} r_{21}\right)^{\times} \underline{\underline{B}}_{2} \Gamma_{1} & 0 & 0 & 0 & \underline{\underline{N}} \\
\left.\underline{\underline{\underline{N}}} \beta_{3}\right)^{\times} \underline{\underline{B}}_{1} & 0 & \left.\underline{\underline{\underline{N}}} r_{32}\right)^{\times} \underline{\underline{B}}_{3} \Gamma_{2} & 0 & 0 & \underline{\underline{N}} \\
\left.\underline{\underline{\underline{N}}} \beta_{4}\right)^{\times} \underline{\underline{B}}_{1} & 0 & \left.\underline{\underline{\underline{N}}} r_{42}\right)^{\times} \underline{\underline{B}}_{3} \Gamma_{2} & \left(\underline{\underline{N}} r_{43}\right)^{\times} \underline{\underline{B}}_{4} \Gamma_{3} & 0 & \underline{\underline{N}} \\
\left(\underline{\underline{\underline{N}}} \beta_{5}\right)^{\times} \underline{\underline{B}}_{1} & 0 & \left.\underline{\underline{\underline{N}}} r_{52}\right)^{\times} \underline{\underline{B}}_{3} \Gamma_{2} & 0 & \left.\underline{\underline{N}} r_{54}\right)^{\times} \underline{\underline{B}}_{5} \Gamma_{4} & \underline{\underline{N}}
\end{array}\right]\left\{\begin{array}{c}
\omega_{1} \\
\sigma_{1} \\
\sigma_{2} \\
\sigma_{3} \\
\sigma_{4} \\
v_{1}
\end{array}\right\}
$$


and, clearing basis dyads, we arrive at

$$
\left\{\begin{array}{l}
v_{1} \\
v_{2} \\
v_{3} \\
v_{4} \\
v_{5}
\end{array}\right\}=\left[\begin{array}{cccccc}
0 & 0 & 0 & 0 & 0 & U \\
\beta_{2}^{\times} C^{1} & r_{21}^{\times} C^{2} \Gamma_{1} & 0 & 0 & 0 & U \\
\beta_{3}^{\times} C^{1} & 0 & r_{32}^{\times} C^{3} \Gamma_{2} & 0 & 0 & U \\
\beta_{4}^{\times} C^{1} & 0 & r_{42}^{\times} C^{3} \Gamma_{2} & r_{43}^{\times} C^{4} \Gamma_{3} & 0 & U \\
\beta_{5}^{\times} C^{1} & 0 & r_{52}^{\times} C^{3} \Gamma_{2} & 0 & r_{54}^{\times} C^{5} \Gamma_{4} & U
\end{array}\right]\left\{\begin{array}{l}
\omega_{1} \\
\sigma_{1} \\
\sigma_{2} \\
\sigma_{3} \\
\sigma_{4} \\
v_{1}
\end{array}\right\}
$$

Noting the patterns in subscripts and superscripts, we conclude that we may write $V$ by inspection for any tree-topology system. 